\title{
Single-particle detection with micro-/nanocantilevers based sensors
}

\author{
I. Stachiv ${ }^{1}$, C.-W. Yang ${ }^{1}$, I.-S. Hwang ${ }^{1}$, Y.-L.Chen ${ }^{1,2,3}$ \\ 1 Institute of Physics, Academia Sinica, Taipei, Taiwan. \\ ${ }^{2}$ Department of Physics, National Taiwan University, Taipei, Taiwan \\ ${ }^{3}$ Department of Chemistry Engineering, National Tsing-Hua University, Hsinchu, Taiwan \\ stachiv@phys.sinica.edu.tw
}

\begin{abstract}
:
We present a theoretical analysis of micro-/nanocantilever based sensors for detection of a particle in vacuum and in viscous fluid. Ultra-high sensitivity detection in fluid will provide better understanding of realistic behavior of molecules and biomolecules in a native environment. We derive simple expressions for the resonant frequency shift accounting for an attached mass in vacuum and viscous fluid. We show that by detecting the first flexural and torsional modes of the cylindrical cantilever (e.g. carbon nanotube or nanowire) the attached mass and position can be accurately resolved. For other cantilever geometries (i.e. rectangular shape, V-shape, etc.) two flexural and torsional modes are needed to determine the mass and the position of the attached particle.
\end{abstract}

Key words: micro-/nanocantilever, mass sensor, flexural mode, torsional mode, resonant frequency.

\section{Introduction}

Micro-/nanocantilevers have potential application as highly sensitive sensors for biological and macro-molecule detection [1]. A particle attached on cantilever can be detected indirectly by measuring the frequency shift of the cantilever [2]. The resonance frequency shift depends on the attached mass and the position of attachment [3]. We have recently shown that the particle mass and the longitudinal position can be resolved by measuring three consecutive flexural resonant frequencies [4]. This method works well in vacuum or in air, where higher vibrational modes can be easily found.

However once the cantilever is immersed in fluid, most often just first two resonant frequency can be detected [5]. The purpose of this paper is to perform a complete theoretical analysis for the cantilever of an arbitrary shape operating as mass sensor in vacuum and viscous fluid and to suggest a high sensitive method for the mass and position detection in fluid.

\section{Theory}

A classical beam theory is used and model is developed for a cantilever of an arbitrary cross sectional area but which is uniform along the entire cantilever length. The fluid, in which the cantilever operates, is incompressible. The attached nanoparticle or macro-molecule is modeled as a point mass $\left(m_{p}\right)$ with a moment of inertia $J_{0}\left(J_{0} \approx m_{p}\left[\left(T_{c} / 2\right)^{2}+y^{2}\right]\right)$ attached on the cantilever, as illustrated in Fig. 1.

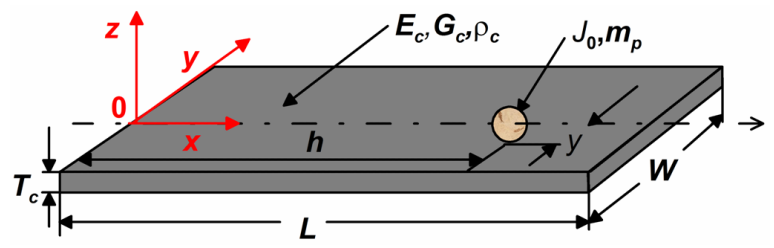

Fig. 1. The sketch of the cantilever with attached nanoparticle

\section{Flexural modes}

The flexural oscillations of the micro/nanocantilever in fluid are described by [6]

$a_{c}^{2} w_{x x x x}+w_{t t}=F_{\mathrm{D}}+F_{\mathrm{M}}$,

where $a_{c}=\left[E_{c} I_{c} /\left(\rho_{c} A_{c}\right)\right]^{0.5}, w, E_{c}, I_{c}, \rho_{c}$, and $L$ are the flexural displacement, the elastic moduli, the second moment of inertia, the density and the length of cantilever, respectively; $F_{\mathrm{D}}$, and $F_{\mathrm{M}}$ are the driving force per unit length and hydrodynamic force of the surrounding fluid per unit length. To close the statement of the problem the following boundary conditions are imposed: 
$\left.w\right|_{0}=\left.w_{x}\right|_{0}=\left.w_{x x}\right|_{L}=\left.w_{x x x}\right|_{L} 0$,

The attached mass is accounted for through the so-called "matching conditions" at the position of the attachment [4]:

$\left.w\right|_{h_{-}}=\left.w\right|_{h_{+}},\left.w_{x}\right|_{h_{-}}=\left.w_{x}\right|_{h_{+}},\left.w_{x x}\right|_{h_{-}}=\left.w_{x x}\right|_{h_{+}}$,

$E_{c} I_{c}\left[\left.w_{x x x}\right|_{h_{-}}-\left.w_{x x x}\right|_{h_{+}}\right]=\left.m_{p} w_{t t}\right|_{h}$,

where $h_{-}$and $h_{+}$denote the location to the left and to the right of the particle.

Solving Eq. (1) in absence of the driving and hydrodynamic forces gives the flexural resonant frequencies in vacuum [4]:

$f_{f n}=\frac{\gamma_{n}^{2}}{2 \pi L^{2}} a_{c}, n=1,2, \ldots$,

$2(\cosh \gamma \cos \gamma-1)-\varepsilon \gamma\left\{F\left[\gamma\left(1-h^{*}\right)\right]-F\left(\gamma h^{*}\right)\right.$

$-\sinh \gamma \cos \gamma h^{*} \cos \left[\gamma\left(1-h^{*}\right)\right]+\cosh \left[\gamma\left(1-h^{*}\right)\right]$

$\left.\times \cosh \gamma h^{*} \sin \gamma\right\}=0$,

where $F(z)=\sinh z \cos z-\cosh z \sin z, \varepsilon=$ $m_{p} / M$ is the mass ratio, $h^{*}=h / L$, and $M$ is the mass of the cantilever.

For small mass ratios $(\varepsilon<1)$, the flexural frequency shift caused by an attached mass can be found by perturbation method [7]. They are given by [4]

$\Delta f_{f_{n}} / f_{f_{n}}=2 \varepsilon \alpha_{f}\left(h^{*}, \gamma_{0 n}\right)$,

where $\alpha_{f}\left(h^{*}, Y_{0 n}\right)=\left\{F\left(Y_{0 n} h^{*}\right)-F\left[\gamma_{0 n}\left(1-h^{*}\right)\right]+\right.$ $\sinh Y_{0 n} \cos \left(Y_{0 n} h^{*}\right) \cos \left[Y_{0 n}\left(1-h^{*}\right)\right]-\sin Y_{0 n}$ $\left.\cosh \left(\gamma_{0 n} h^{*}\right) \cosh \left[\gamma_{0 n}\left(1-h^{*}\right)\right]\right\} / 2 F\left(\gamma_{0 n}\right)$ and $\gamma_{01}=$ $1.875 \ldots, Y_{02}=4.694 \ldots$. . The functions $\alpha_{f}\left(h^{*}\right.$, $\left.Y_{01}\right)$ and $\alpha_{f}\left(h, \gamma_{02}\right)$ are shown in Fig. 2.

\section{Torsional modes}

The torsional oscillations of the micro/nanocantilever in fluid are known [6] and read

$c_{c}^{2} \varphi_{x x}-\varphi_{t t}=T_{\mathrm{D}}+T_{\mathrm{M}}$,

where $c_{c}=\left[G_{c} J /\left(\rho_{c} I_{P c}\right)\right]^{0.5}, \varphi, G_{c}, J, I_{p c}$, are the rotating angle, the shear moduli, the torsional constant, the polar moment of inertia. $T_{\mathrm{D}}$ and $T_{\mathrm{M}}$ are the driving and surrounding fluid torques per unit lengths. Similarly to the flexural modes, the following boundary and matching conditions are imposed:

$\left.\varphi\right|_{0}=\left.\varphi_{x}\right|_{L}=0$

and

$\left.\varphi\right|_{h_{-}}=\left.\varphi\right|_{h_{+}}, G J_{c}\left[\left.\varphi_{x}\right|_{h_{+}}-\left.\varphi\right|_{h_{-}}\right]=\left.J_{0} \varphi_{t t}\right|_{h}$.
Analogous to the flexural modes, the torsional frequency shifts in vacuum due to the attached mass are given by

$f_{\text {tn }}=\frac{v_{n}}{2 \pi L} c_{c}, n=1,2, \ldots$,

$\cos v-\varepsilon \frac{T_{c}^{2}+4 y^{2}}{T_{c}^{2}+W^{2}} v \sin v h^{*} \cos \left[v\left(1-h^{*}\right)\right]=0$.

Accounting for $\varepsilon<1$, the torsional frequency shifts due to the attached mass can be expressed through the unloaded cantilever as

$\Delta f_{t_{n}} / f_{t_{n}}=3 \varepsilon \frac{T_{c}^{2}+4 y^{2}}{T_{c}^{2}+W^{2}} \alpha_{t}\left(v_{0 n}, h^{*}\right)$,

where $\alpha_{t}\left(h^{*}, v_{0 n}\right)=(-1)^{n+1} \sin \left(v_{0 n} h^{*}\right) \cos \left[v_{0 n}(1-\right.$ $\left.\left.h^{*}\right)\right], \quad v_{0}=(2 n-1) \pi / 2$ and $y \in\left\langle 0, \frac{W}{2}\right\rangle$. The functions $\alpha_{t}\left(h^{*}, v_{01}\right)$ and $\alpha_{t}\left(h^{*}, v_{02}\right)$ are also given in Fig. 3.

It should be emphasized here that in fluid, $f_{f n}$ and $f_{t n}$ are just the flexural and torsional resonant frequencies in fluid, respectively [6].

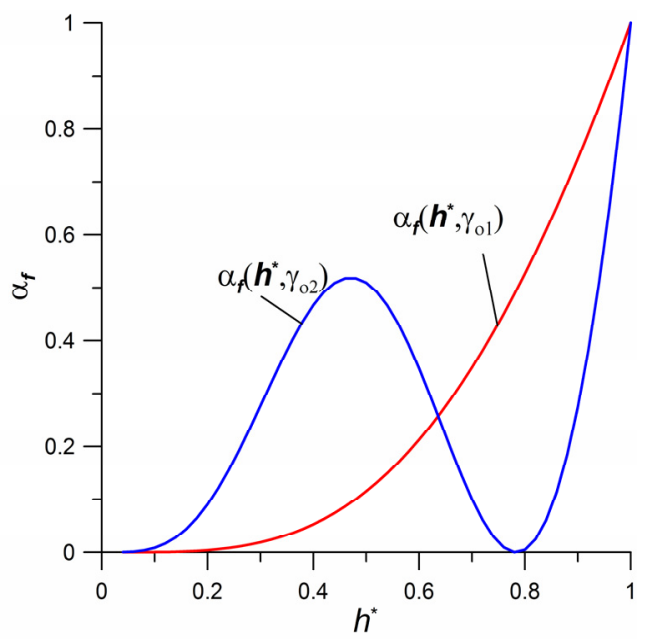

Fig. 2. The variation of the functions $\alpha_{f}\left(h^{*}, Y_{01}\right)$ and $\alpha_{f}\left(h^{*}, y_{02}\right)$ for various $h^{*}$.

\section{Determining attached mass and position}

The frequency shifts given by Eqs. (6) and (12) depend on the mass ratio $\varepsilon$ and the position of attachment through coefficients $\alpha$. It is evident from Figs. 2 and 3 that for a given particle mass, the first and second vibrational modes are different from each other. This allows one to resolve mass and position by considering the ratios $\Gamma_{f}=\alpha_{f}\left(h^{*}, \gamma_{02}\right) / \alpha_{f}\left(h^{*}, \gamma_{01}\right)$ and $\Gamma_{t}=$ $\alpha_{t}\left(h^{\star}, v_{02}\right) / \alpha_{t}\left(h^{*}, v_{01}\right)$ as is shown in Fig. 4. 


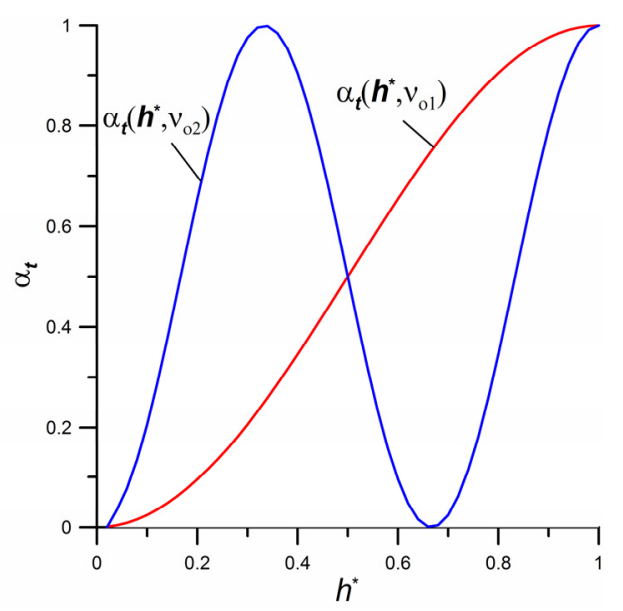

Fig. 3. The variation of the functions $\alpha_{t}\left(h^{*}, v_{01}\right)$ and $\alpha_{t}\left(h^{*}, v_{02}\right)$ for various $h^{*}$.

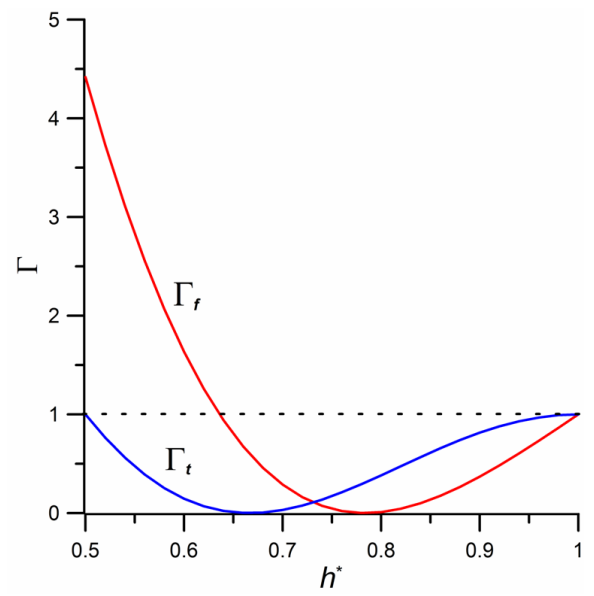

Fig. 4. Ratios of the torsional $\left(\Gamma_{t}\right)$ and flexural modes $\left(\Gamma_{f}\right)$ for different positions, respectively.

For the same particle position the ratios of $\Gamma_{f}$ and $\Gamma_{t}$ are different from each other. Hence the molecule mass and position can be determined. As seen in Fig. 4, two cases can be realized: i) $\Gamma_{f}>1$, where two flexural and one torsional modes allows one to determine particle mass and position; ii) $\Gamma_{f} \leq 1$, where the second torsional mode is needed. Hence the mass and position can be determined. As an example we consider $\Delta f_{f_{1}} / f_{f 1}=\Delta f_{f 2} / f_{f 2}=0.01$ and $\Delta f_{t 1} / f_{t 1}=$ $\Delta f_{t 2} / f_{t 2}=0.1$. From the flexural modes, two possible molecule position of $h^{\star}=0.636$ or 1 can be obtained (see Fig. 2). Since $\Gamma_{t}=1$ only at $h^{*}=1$, the particle position and the coefficient $\alpha_{f}\left(1, \gamma_{01}\right)=1$ can be resolved. By substituting the value of coefficient $\alpha_{f}$ into the Eq. (6), the desired mass ratio of $\varepsilon_{f}=0.005$ is found. Substituting the determined values of the attached mass $\left(m_{p}\right)$ and longitudinal position $(h)$ into the Eq. (12) and accounting for $\Delta f_{t 1} / f_{t 1}=0.1$ allows us as well to find the two possible $y$ locations of the attached particle.
The above analysis becomes much easier when the cantilever has a cylinder shape with a diameter of $D$ (e.g. carbon nanotube or nanowire). Then moments of inertia of the single-particle and cantilever are given by

$J_{0} \approx m_{p}(D / 2)^{2}$,

$J_{c} \approx \rho_{c} L \pi D^{4} / 32$,

and consequently, the frequency shift given by Eq. (12) yields

$\Delta f_{t_{n}} / f_{t_{n}}=2 \varepsilon \alpha_{t}\left(h^{*}, v_{0 n}\right)$.

As can be seen from Eqs. (6) and (15), the frequency shifts for both the flexural and torsional modes depend only on the mass ratio $\varepsilon$ and the position of attachment through coefficients $\alpha$. We emphasis here that these coefficients for first flexural and torsional mode are different from each other as shown in Fig. 5. Introducing the ratio between torsional and flexural mode $\Gamma$ (see Inset of Fig. 5) as

$$
\Gamma=\alpha_{t}\left(h^{*}, v_{01}\right) / \alpha_{f}\left(h^{*}, \gamma_{01}\right) \text {, }
$$

the mass and position of the attachment can be resolved from measurement of only the first flexural and torsional modes. As an example suppose that the measured frequency ratios are $\Delta f_{f 1} / f_{f 1}=0.1$ and $\Delta f_{t 1} / f_{t 1}=0.01$. By using the Eq. (16) and inset of Fig. 5, the position of the attachment $h^{*}=0.5$ is found. From Fig. 5, the coefficients $\alpha$ (e.g. $\alpha_{t}=0.4$ ) are obtained, and consequently, the mass ratio $\varepsilon=0.013$ is found.

\section{Conclusions}

We derived simple analytical formulas for the frequency shift of the cantilever operating in flexural and torsional mode caused by an attached particle (e.g. macro-molecule, gold nanoparticle, etc.) at an arbitrary location. We show that the mass and position of the attachment can be resolved for an arbitrary shape of the cantilever by measuring the first two flexural and torsional modes. In case of the cylindrical cantilever the mass and position can be determined by using only the first flexural and torsional modes.

\section{Acknowledgements}

This work was supported by the Academia Sinica Career Development Award, No. 100CD4-M01 and the National Science Council of Taiwan, NSC 98-2112-M-001-004-MY3. 


\section{References}

[1] Kim, D. Yi, A. Passian, T. Thundat, Applied Physics Letters 96, 153703-1-153703-3 (2010); doi:10.1063/1.3399234

[2] K. Jensen, K. Kim, A. Zettl, Nature Nanotechnology 3, 533-537 (2008); doi:10.1038/nnano.2008.200

[3] C. Dohn, W. Svensen, A. Boisen, O. Hansen, Review of Scientific Instruments 78, 103303-1103303-3 (2007); doi:10.1063/1.2804074

[4] I. Stachiv, A. I. Fedorchenko, Y.-L. Chen, Applied Physics Letters 100, 093110-1-093110-3 (2012); doi:10.1063/1.3691195

[5] S. Sawano, T. Arie, S. Akita, Nano Letters 10, 3395-3398 (2010); doi: 10.1021/nl101292b

[6] C. A. Van Eysden, J. E. Sader, Journal of Applied Physics 101, 044908-1-044908-11 (2007); doi:10.1063/1.2654274

[7] A. H. Nayfeh, Perturbation method, (WILEY-VCH Verlag $\mathrm{GmbH} \&$ CO. Weinheim, 2004); doi.wiley.com/10.1002/9783527617609.fmatter 